\title{
La usabilidad en los sistemas de inteligencia de negocios, un caso práctico
}

\section{Usability in business intelligence systems, a case study}

\author{
Margarita Alejandra Aucancela Guamán. ${ }^{1}$ \\ Recibido: 20-06-2019 / Revisado: 27-07-209 /Aceptado: 17-08-2019/ Publicado: 06-09-2019
}

\begin{abstract}
DOI: https://doi.org/10.33262/cienciadigital.v3i3.3.824

The usability of business intelligence systems is a fundamental acceptance test of the software, so it is important not only to reduce errors but also to reduce costs in maintenance and resources, which is why in this research work the usability of a business intelligence system developed for the generation of evaluation and academic management indicators was evaluated for which a survey was applied to teachers in charge of the processes of career self-evaluation, as a result it has to be more than $70 \%$ of Users are satisfied with the system. Usability tests are fundamental when evaluating the acceptance of a software Keywords: usability, business intelligence, satisfaction

\section{Resumen}

La usabilidad de los sistemas de inteligencia de negocios se constituye en una prueba de aceptación fundamental del software por lo que es importante realizarla no solo para reducir errores sino también para reducir costes en mantenimiento y recursos, es por ello que en el presente trabajo de investigación se evaluó la usabilidad de un sistema de inteligencia de negocios desarrollado para la generación de indicadores de evaluación y gestión académica para lo cual se aplicó una encuesta a docentes encargados de los procesos de autoevaluación de carreras, como resultado se tiene que más del $70 \%$ de los usuarios se encuentran satisfechos con el sistema. Las pruebas de usabilidad son fundamentales al momento de evaluar la aceptación de un software.

Palabras claves: Usabilidad, Inteligencia de Negocios, Satisfacción
\end{abstract}

\section{Introducción}

La inteligencia de negocios se define con el conjunto de metodologías, aplicaciones, prácticas y capacidades enfocadas a la creación y administración de información que permite tomar mejores decisiones a los usuarios de una organización (Curto, 2016). Incluye las aplicaciones, la infraestructura, las herramientas, y las mejores prácticas que permiten el acceso y el análisis de la información para mejorar y optimizar las decisiones y el rendimiento de las organizaciones (Gartner, 2017)

Los sistemas de inteligencia de negocios facilitan la presentación de informes y análisis de datos que son utilizados para la toma de decisiones, conocer por ejemplo en una universidad el rendimiento académico de los estudiantes, o saber el número de aprobados para planificar el siguiente periodo académico son datos importantes. Cabe mencionar que la información presentada por los sistemas de inteligencia de negocios es de utilidad para los gestores académicos sean estos: rectores, vicerectores, directores de carrera y personal administrativo como secretarias académicas entre otros, por lo que es importante evaluar la usabilidad de un

\footnotetext{
1 Escuela Superior Politécnica de Chimborazo, maucancela@espoch.edu.ec
} 
sistema de inteligencia de negocios.

Una de las fases más importantes en el desarrollo del software, háblese en este caso de los sistemas de inteligencia de negocios, es la fase de pruebas debido a que las mismas pueden consumir hasta el $50 \%$ del coste total del sistema y este porcentaje es mayor cuando se trata de sistemas críticos.

Las pruebas tienen como objetivos eliminar todos los errores que puedan aparecer en la mínima cantidad de tiempo y esfuerzo, a la vez que buscan incrementar la confianza en los usuarios en que el sistema cumple con los requisitos deseados. Las pruebas en el desarrollo de software pueden ser unitarias, de integración, de sistema y de aceptación.

Las pruebas unitarias están diseñadas para probar una parte pequeña y específica de funcionalidad de un sistema, las pruebas de integración están diseñadas para probar la interacción entre los distintos componentes de un sistema. Las pruebas de sistema se encuentran diseñadas para probar el sistema en su totalidad como si de una caja negra se tratase y las pruebas de aceptación están diseñadas para verificar que el sistema cumple con los requisitos exigidos por el usuario.

Las pruebas de aceptación sirven para establecer el grado de adherencia a las necesidades, requerimientos y procesos de negocio solicitados por el usuario de un sistema, en función de estas pruebas el usuario decide si acepta o no el sistema que le está siendo entregado.

Las pruebas de usabilidad es una fase crítica del proceso de diseño y desarrollo centrado en el usuario, donde los equipos de productos pueden observar y medir la usabilidad de la funcionalidad, las interacciones y las interfaces de usuario de sus soluciones de software (Germanakos \& Fichte, 2019)

La usabilidad gana importancia debido a que genera una reducción de los costos evitando el sobre diseño en el desarrollo de un sistema, disminuye el número de mantenimientos y reduce el tiempo dedicado al soporte a usuarios (Conexión ESAN, 2015). El manejo de la usabilidad en una etapa temprana, lo que reduce el consumo de tiempo, esfuerzos y recursos (Lina A. Hasan, 2015)

Por otro lado, la usabilidad se define como la medida de la calidad de la experiencia que tiene un usuario cuando interactúa con un sistema, para determinar que un sistema sea fácil de usar y aprender, sea eficiente en el uso de los elementos ofrecidos en pantalla y sea efectivo en el cumplimiento de las tareas que se pueden llevar a cabo a través de ella (Sánchez, 2011)

Dentro de los factores que determinan la usabilidad podemos mencionar la accesibilidad, legibilidad, navegabilidad, facilidad de aprendizaje, velocidad de utilización, eficiencia del usuario y tasas de error (Sánchez, 2011). Según (Poropat, 2014), los factores que evalúan la usabilidad en los sistemas de inteligencia de negocios son: facilidad de aprendizaje del sistema, la eficiencia del sistema, la flexibilidad del sistema, la participación de los usuarios del sistema y la satisfacción del usuario.

En relación, a los atributos, se tiene:

- Flexibilidad del sistema: Hace referencia proporcionar la capacidad de ampliar o cambiar las funcionalidades existentes de una manera adecuada y conveniente (Lina A. Hasan, 2015)

- Facilidad de aprendizaje del sistema: es una parte de la eficacia. Es la capacidad del usuario para operar el sistema a un cierto nivel de competencia definido después de un periodo definido de entrenamiento. (Poropat, 2014)

- Eficiencia del sistema: Evalúa en qué medida un usuario específico consume recursos con el fin de lograr sus objetivos con eficacia.

- Participación de los usuarios del sistema: es una cualidad de la experiencia del 
usuario con la tecnología que depende de la estética, la novedad y la facilidad de uso del sistema. Es la capacidad del usuario para atender y participar en la experiencia, y la evaluación general del usuario de la experiencia (Poropat, 2014).

- Satisfacción de los usuarios: Refleja el nivel de satisfacción de los usuarios a utilizar el sistema en un contexto particular (Lina A. Hasan, 2015). Evalúa la forma en que el usuario se sienta sobre el sistema y cuáles son sus opiniones, en el caso de los sistemas de inteligencia de negocios se en que grado afecta la capacidad de uso del sistema de BI.

\section{Metodología}

En el presente trabajo de investigación, se aplicó un enfoque cuantitativo, siguiendo la metodología propuesta por (Sampieri, 2010). Para desarrollar el planteamiento del problema y la perspectiva teórica se realizó una revisión bibliográfica de artículos y publicaciones relacionadas con las pruebas de aceptación, las pruebas de usabilidad y la inteligencia de negocios, las bases científicas consultadas fueron de Springer, ScienceDirect, IEEE y ACM. Posteriormente se diseño una encuesta para evaluar la usabilidad de un sistema de inteligencia de negocios denominado: sistema de gestión de indicadores de evaluación académica. La encuesta se aplicó a 20 docentes encargados de llevar los procesos de autoevaluación de carreras.

\section{Resultados}

A continuación, se tiene los resultados de la encuesta aplicada a usuarios del sistema de inteligencia de negocios desarrollado para obtener indicadores de evaluación y gestión académica, en la encuesta se evaluación la usabilidad del sistema en relación a la facilidad de aprendizaje del sistema, la eficiencia del sistema, la flexibilidad del sistema, la participación de los usuarios del sistema y la usabilidad del sistema.

\section{Facilidad de Aprendizaje del Sistema}

1. En la pregunta relacionada sobre si el sistema al ser utilizado cubre la mayoría de las respuestas a las preguntas que aparecen, se tiene los siguientes resultados:

20 respuestas
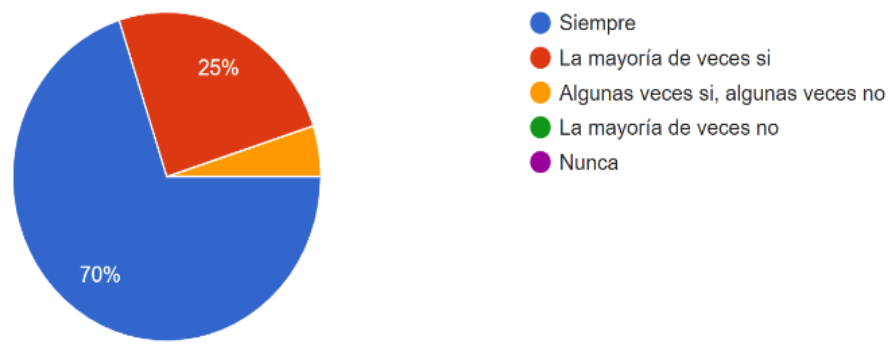

Figura 1. Respuesta que el sistema presenta frente a preguntas del usuario

Esto demuestra que, del total de encuestados, el 70\% respondió que el sistema siempre responde a las preguntas que aparecen al momento de ser utilizado, un $25 \%$ responde a que el sistema responde la mayoría de las veces a las preguntas planteadas por el usuario y un 5\% seleccionó, que el sistema algunas veces si responde y algunas veces no lo hace.

2. La capacitación dada sobre el uso del sistema permite la gestión rápida y eficaz de la información, así como la ayuda en el aprendizaje de sus características 


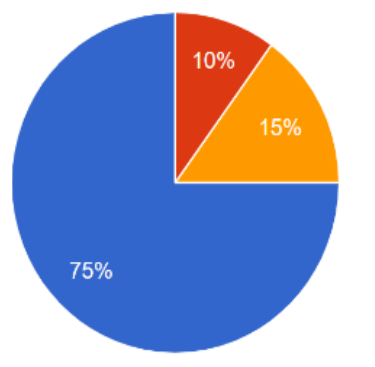

Figura 2. Resultado de la gestión rápida y eficaz del sistema

Esto determina que, del total de encuestados, el 75\% respondió que la capacitación permite la gestión rápida y eficaz de la información, mientras que el 10\% mencionó que la mayoría de las veces si y el 15\% algunas veces si y otras veces no.

Considerando la respuesta de los encuestados en relación a la facilidad de aprendizaje del sistema, se determinó que más del 70\% de los encuestados, están de acuerdo con el mismo.

\section{Eficiencia del Sistema}

3. El sistema apoya plenamente en el desarrollo de las actividades que usted realiza

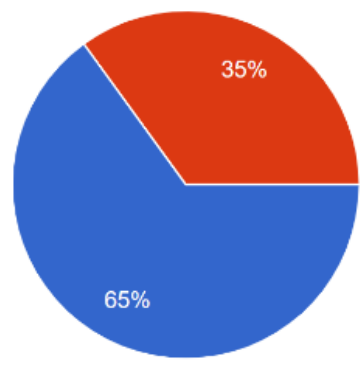

Siempre

La mayoria de veces si

Algunas veces si, algunas veces no

La mayoria de veces no

- Nunca

Figura 3. Apoya el sistema al desarrollo de las actividades del usuario

Del total de encuestados, se tiene que el $65 \%$ respondió a que el sistema siempre apoya al desarrollo de las actividades que realiza, mientras que el 35\% restante respondió que el sistema apoya la mayoría de las veces.

4. A su criterio, el sistema contribuye a generar los indicadores de evaluación necesarios para el proceso en el cual usted se desempeña, por ejemplo: se obtiene el número de publicaciones por docente, por periodo académico 


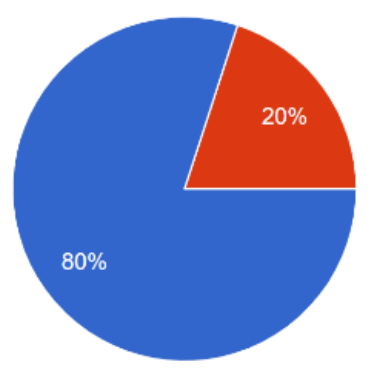

Siempre

La mayoría de veces si

Algunas veces si, algunas veces no

La mayoría de veces no

Nunca

Figura 4. Contribuye el sistema a generar los indicadores de evaluación

En este apartado, se tiene que el $80 \%$ de los encuestados, ratifican que el sistema contribuye al proceso en el cual se desempeñan los miembros de la comisión de evaluación, mientras que el $20 \%$ mencionó que la mayoría de las veces si contribuye a generar indicadores de evaluación.

En las preguntas planteadas para evaluar la eficiencia del sistema, se tiene que el sistema apoya y contribuye a la generación de indicadores de evaluación, necesarios para los procesos de autoevaluación de las carreras de la ESPOCH

Flexibilidad del Sistema

5. El sistema tiene funcionalidades integradas que pueden ser configuradas de acuerdo a sus necesidades.

Por ejemplo: Permite generar reportes en otros formatos, Permite cambiar los cubos de información, Permite generar gráficas que resumen la información, entre otros

20 respuestas
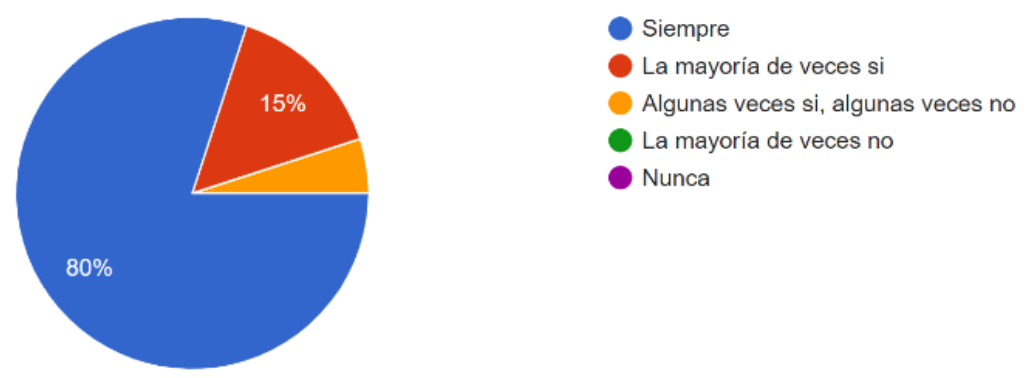

Figura 5. Se configura el sistema de acuerdo con las necesidades del usuario

En relación a esta pregunta, se tiene que en el 80\% de los encuestados se encuentran de acuerdo con las funcionalidades que posee el sistema, mientras que el $15 \%$ respondió la mayoría de las veces si, mientras que el $5 \%$ respondió que algunas veces si, algunas veces no. 
6. Usted tiene acceso al sistema en cualquier lugar donde precise de información
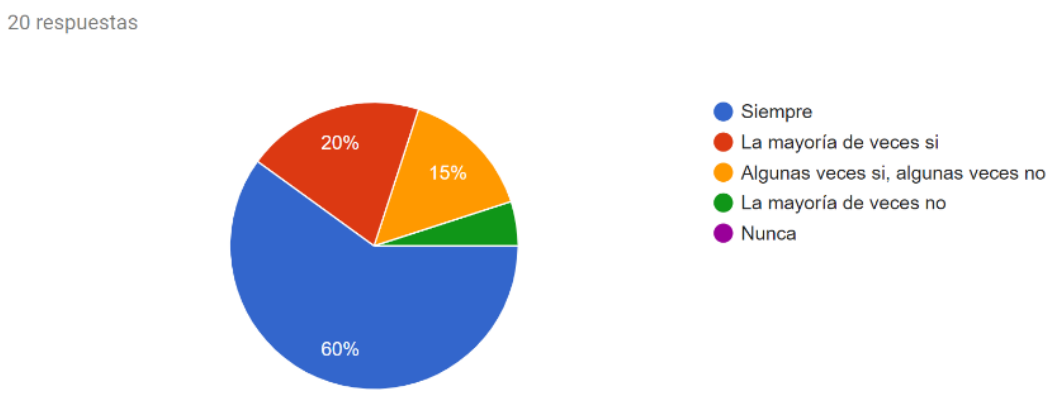

Figura 6. Acceso al sistema en cualquier lugar

Del total de encuestados, se tiene que el $60 \%$ mencionó que tiene siempre acceso al sistema, mientras que el $20 \%$ respondió que la mayoría de las veces si, un 15\% mencionó que algunas veces si, algunas veces no y un 5\% mencionó que la mayoría de las veces no. En relación al 5\% que mencionó que la mayoría de las veces no tiene acceso al sistema, se puede decir que esto es temporal, en vista de que al momento se tiene un prototipo del sistema.

Para sintetizar los resultados, se puede decir que la flexibilidad del sistema es superior al $60 \%$, hay que considerar que al momento el sistema se desarrolló en una determinada facultad y se dispone al momento de un prototipo puesto a prueba.

Participación de los Usuarios

7. ¿Tengo el conocimiento y las habilidades necesarias para utilizar el sistema?

20 respuestas

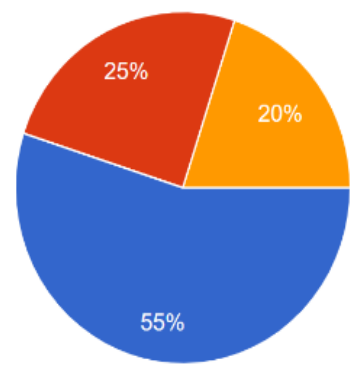

Nunca

Figura 7. Tiene el usuario el conocimiento y las habilidades para utilizar el sistema

Del total encuestados, se tiene que en un 55\% poseen siempre el conocimiento y las habilidades necesarias para utilizar el sistema, mientras que un $25 \%$ mencionó que la mayoría de las veces si y un $20 \%$ mencionó que algunas veces si, algunas veces no. 


\section{OClencia

8. Conozco todas las funcionalidades que posee el sistema

\section{0 respuestas}

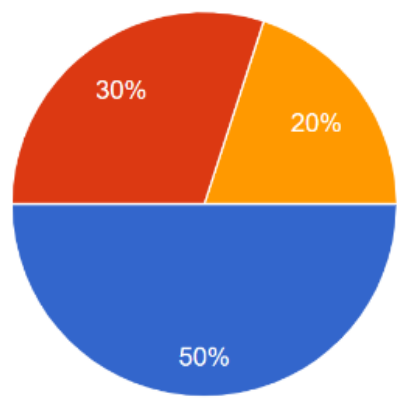

Totalmente de acuerdo

De acuerdo

Neutral

En desacuerdo

Totalmente en desacuerdo

Figura 8. Conoce el usuario todas las funcionalidades del sistema

En relación con el conocimiento de las funcionalidades que posee el sistema, se tiene que el 50\% se encuentra totalmente de acuerdo, mientras que el $30 \%$ se encuentra de acuerdo y un $20 \%$ se muestra neutral al conocimiento de la misma, esto permite determinar que el conocimiento en relación al sistema es progresivo, a medida que se generen más interacciones con el mismo, mejorará su conocimiento.

9. Estoy familiarizado con los procedimientos para alcanzar los objetivos establecidos. Por ejemplo: Conozco el procedimiento para realizar el cálculo de indicadores

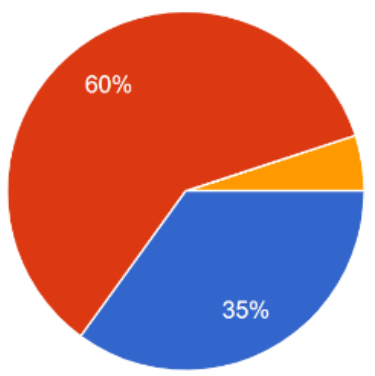

Totalmente de acuerdo

De acuerdo

Neutral

En desacuerdo

Totalmente en desacuerdo

Figura 9. Conoce el usuario los procedimientos para alcanzar los objetivos de evaluación

En relación, al conocimiento del cálculo de indicadores se tiene que, del total de encuestados, el $60 \%$ se encuentra totalmente de acuerdo, mientras que el $35 \%$ se encuentra de acuerdo y un $5 \%$ tiene una posición neutral.

En relación con la participación de los usuarios en los procesos de evaluación y sus objetivos se tiene que más del $80 \%$ de los usuarios participan en los procesos y poseen el conocimiento necesario para utilizar el sistema.

Tareas 
10. Las tareas que se realizan con el sistema, ¿se utilizan habitualmente?

\section{0 respuestas}

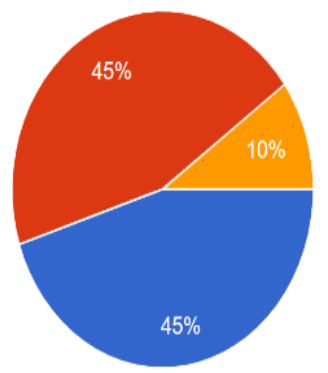

Totalmente de acuerdo

De acuerdo

Neutral

En desacuerdo

Totalmente en desacuerdo

Figura 10. Las tareas que realiza el sistema son habituales para el usuario

Con relación a esta pregunta se tiene que el $45 \%$ de los encuestados, está totalmente de acuerdo con el hecho de que las tareas que se realizan con el sistema son habituales, mientras que el $45 \%$ se encuentra de acuerdo y el $10 \%$ se encuentra en forma neutral.

11. El sistema le permite solucionar alguna tarea complicada

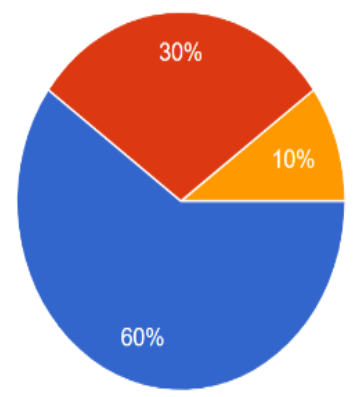

Figura 11. Soluciona el sistema alguna tarea complicada

En relación con esta pregunta se tiene que el $60 \%$ de los encuestados está totalmente de acuerdo, mientras que el $30 \%$ se encuentra de acuerdo y el 10\% se ubica en una posición neutral.

Al evaluar la respuesta del sistema en relación a las tareas que desarrolla los encuestados se tiene que más del $60 \%$ está totalmente de acuerdo con el apoyo que tiene el sistema al desarrollo de sus tareas, más si estas son complicadas.

Satisfacción del usuario 
12. ¿Cree usted que el sistema es amigable con el usuario?

20 respuestas

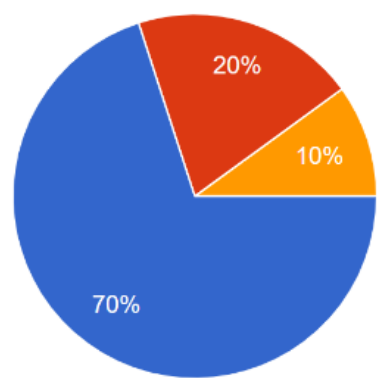

Totalmente de acuerdo

De acuerdo

Neutral

En desacuerdo

Totalmente en desacuerdo

Figura 12. Contribuye el sistema a generar indicadores de evaluación

En esta pregunta, se tiene que el $70 \%$ de los encuestados, se encuentra totalmente de acuerdo con que el sistema es amigable al usuario, un $20 \%$ se encuentra de acuerdo y un $10 \%$ se ubica en una posición neutral.

13. Cree usted que el sistema contribuye al cumplimiento rápido y fácil de tareas y objetivos establecidos

Del total de encuestados se tiene que el $80 \%$ ratifica que el sistema a plataforma contribuye al cumplimiento rápido y fácil de tareas y objetivos establecidos en los procesos de autoevaluación.

20 respuestas

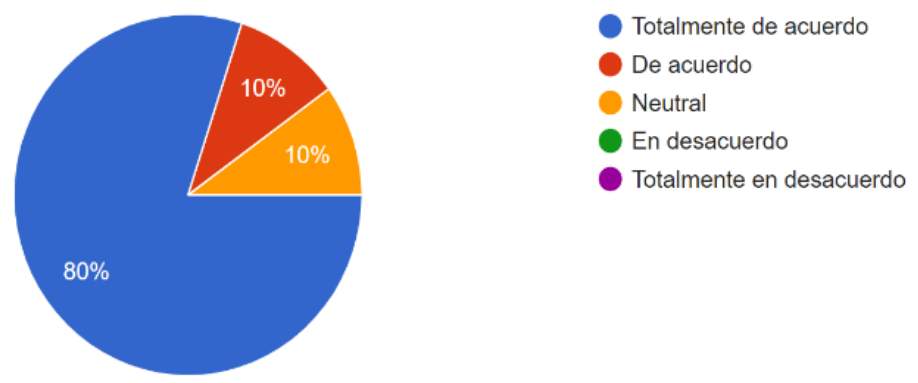

Figura 13. Contribuye el sistema al cumplimiento rápido y fácil de tareas y objetivos

La usabilidad del sistema de gestión de indicadores se vio demostrada al tener más del $70 \%$ de criterios, en los cuales los usuarios mencionaron que se encuentran totalmente de acuerdo en que el sistema es amigable y permite el cumplimiento rápido y fácil de tareas y objetivos establecidos en el proceso. 


\section{Discusión}

Develando los conceptos, se tiene que las pruebas de usabilidad son parte de las pruebas de aceptación del software y su temprana gestión reduce costos, tiempo, esfuerzos, recursos y el número de mantenimientos (Conexión ESAN, 2015) y (Lina A. Hasan, 2015)

En relación con la evaluación de la usabilidad del sistema de inteligencia de negocios se determinó que:

- Más del 70\% de los usuarios encuestados establecieron que el sistema siempre es fácil de aprender.

- En relación con la eficiencia del sistema, se tiene que este apoya y contribuye a la generación de indicadores de evaluación y de gestión académica.

- En relación con la flexibilidad del sistema, se tiene que el mismo es superior al $60 \%$, cabe recalcar que al momento el sistema se desarrolló para una determinada dependencia.

- En relación con la participación de los usuarios en los procesos de evaluación y sus objetivos, se tiene que más del $80 \%$ de los usuarios participan en los procesos y poseen el conocimiento necesario para utilizar el sistema.

- Al evaluar la respuesta del sistema en relación con las tareas que desarrolla los encuestados se tiene que más del $60 \%$ está totalmente de acuerdo con el apoyo que tiene el sistema al desarrollo de sus tareas, más si estas son complicadas

- En relación con la satisfacción de los usuarios del sistema de gestión de indicadores se vio demostrada al tener más del $70 \%$ de criterios, en los cuales los usuarios mencionaron que se encuentran totalmente de acuerdo en que el sistema es amigable y permite el cumplimiento rápido y fácil de tareas y objetivos establecidos en los procesos de evaluación

\section{Conclusión}

La usabilidad determina la aceptación de un software por parte de los usuarios, en el caso práctico al aplicar la encuesta se tiene que la mayoría de las respuestas en relación con los atributos de usabilidad evaluados supera más del $50 \%$ de usuarios encuestados, lo que quiere decir que el sistema de inteligencia de negocios es aceptado por parte de los usuarios.

Un sistema de inteligencia de negocios puede ser evaluado como un software, por lo que no hay diferencias significativas en los atributos de usabilidad empleados al momento de aplicar las pruebas de aceptación del software.

\section{Referencias}

Alter, M. (2012). WIEGO. Retrieved from La economía informal: definiciones, teorías y políticas: http://www.wiego.org/sites/default/files/publications/files/Chen-InformalEconomy-Definitions-WIEGO-WP1-Espanol.pdf

Argos, M. (2014). EL COMERCIO INFORMAL Y SU INCIDENCIA EN LAS VENTAS DE LOS COMERCIANTES FORMALES DEL CENTRO COMERCIAL POPULAR "LA CONDAMINE” SECCIÓN PRENDAS DE VESTIR DURANTE EL PERÍODO 2014. Retrieved from http://dspace.unach.edu.ec/handle/51000/3887

Conexión ESAN. (2015). Retrieved from ¿Por qué la usabilidad es tan importante en una plataforma digital?: https://www.esan.edu.pe/apuntes-empresariales/2015/09/por-queusabilidad-tan-importante-plataforma-digital/ 
Curto, J. (2016). Introducción al business intelligence. Editorial UOC.

El Telégrafo. (2014). Regional Centro. Retrieved from El mercado La Merced, en Riobamba, renueva su imagen: https://www.eltelegrafo.com.ec/noticias/regional/1/el-mercado-lamerced-en-riobamba-renueva-su-imagen

Gartner. (2017). Retrieved from IT Glossary: https://www.gartner.com/it-glossary/

Germanakos, P., \& Fichte, L. (2019). Un método y una herramienta para analizar datos de pruebas de usabilidad en el sector empresarial. INTERACT 2019, 584-588.

INEC. (2018, 03). Reporte de Economía Laboral. Retrieved from http://www.ecuadorencifras.gob.ec/documentos/web-inec/EMPLEO/2018/Marzo2018/Informe_Economia_laboral-mar18.pdf

Lina A. Hasan, K. T.-S. (2015). An Integrated Measurement Model for Evaluating Usability Attributes. New York: Conferencia Internacional sobre Procesamiento Inteligente de Información, Seguridad y Comunicación Avanzada.

López, P. (2015). Nexos. Retrieved from Del comercio informal y los espacios públicos: https://labrujula.nexos.com.mx/?p=309

Poropat, E. (2014). Evaluation of Business Intelligence System Usability. Ljubljana.

Revista Líderes. (2015). Revista Líderes. Retrieved from La informalidad laboral, una condición arraigada en Ecuador: https://www.revistalideres.ec/lideres/informalidadlaboral-condicion-ecuador.html

Romero, D. (2014). DISEÑO DE UN SISTEMA DE MANEJO INTEGRAL DE RESIDUOS SÓLIDOS EN EL MERCADO LA ESPERANZA, CIUDAD DE RIOBAMBA, PROVINCIA DE CHIMBORAZO, MARZO - DICIEMBRE 2014. Riobamba: ESPOCH.

Sampieri, R. (2010). Metodología de la Investigación. México: McGRAW-HILL.

Sánchez, W. (2011). Retrieved from La usabilidad en Ingeniería de Software: Definición y Características: https://core.ac.uk/download/pdf/47264961.pdf

\section{$\lfloor$ Ciencia}




\section{PARA CITAR EL ARTÍCULO INDEXADO.}

Aucancela Guamán, M. (2019). La usabilidad en los sistemas de inteligencia de negocios, un caso práctico. Ciencia Digital, 3(3.3), 319-330.

https://doi.org/10.33262/cienciadigital.v3i3.3.824

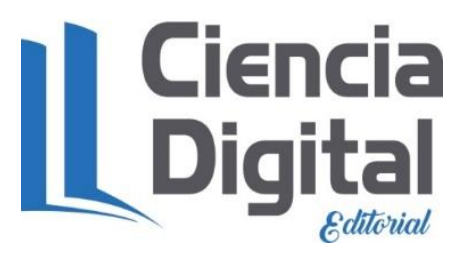

El artículo que se publica es de exclusiva responsabilidad de los autores y no necesariamente reflejan el pensamiento de la Revista Ciencia Digital.

El artículo queda en propiedad de la revista y, por tanto, su publicación parcial y/o total en otro medio tiene que ser autorizado por el director de la Revista Ciencia Digital.
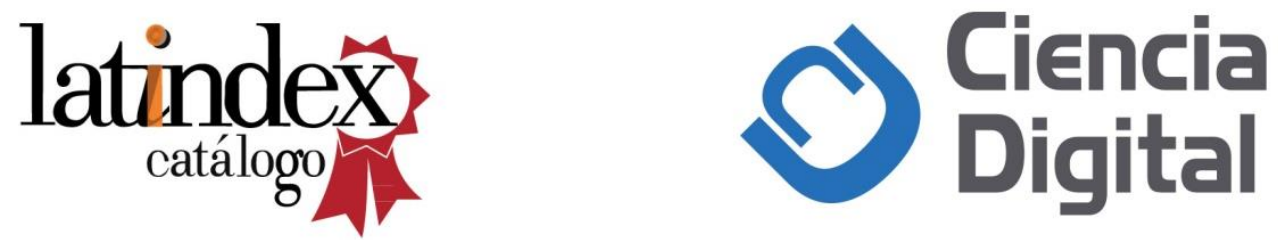